\title{
Simultaneous rapid detection of Hantaan virus and Seoul virus using $R T$-LAMP in rats
}

\author{
Xin Sui ${ }^{1,2}$, Xu Zhang ${ }^{1,3}$, Dongliang Fei ${ }^{1}$, Zhen Zhang ${ }^{3}$, Mingxiao Ma ${ }^{\text {corresp. } 1}$ \\ 1 Institute of Biological Sciences, Jinzhou Medical University, Jinzhou, Liaoning, China \\ 2 The First Affiliated Hospital, Jinzhou Medical University, Jinzhou, Liaoning, China \\ 3 Microbiological laboratory, Center for Disease Control and Prevention of Jinzhou, Jinzhou, Liaoning, China \\ Corresponding Author: Mingxiao Ma \\ Email address: mamingxiao@jzmu.edu.cn
}

Background. Hemorrhagic fever with renal syndrome (HFRS) is in most cases caused by the Hantaan virus (HTNV) and Seoul virus (SEOV). To develop and apply Reverse Transcription Loop-mediated Isothermal Amplification (RT-LAMP) to detect Hantaan virus (HTNV) and Seoul virus (SEOV) simultaneously, which was faster, more cost effective, and easier to perform as the target gene amplified rapidly. In this article an assay based on LAMP is demonstrated, which only employs such apparatus as a water bath or a heat block. Methods. A chromogenic method using the calcein/ $\mathrm{Mn}^{2+}$ complex and real-time turbidity monitoring method were used to assess reaction progress of the reaction, and the specificity of the RT-LAMP-based assay was assessed by detecting cDNAs/cRNAs generated from Coxsackievirus A16 (CA16), Influenza virus (IV), Lymphocytic Choriomeningitis Virus (LCM), mouse poxvirus (MPV), Rotavirus (RV), Mouse hepatitis virus (MHV). In addition, twenty-three clinical specimens were used to determine the agreement between the RTLAMP assay with RT-PCR and immunofluorescence (IFT) method. Results. The detection limit of RT-LAMP to HNTV and SEOV was as low as 10 copies/ $\mu \mathrm{L}$ with optimized reaction conditions, which was much more sensitive than the RT-PCR method (100-1000 copies $/ \mu \mathrm{L}$ ). At the same time, the detection results of twenty-three clinical specimens have also illustrated the agreement between this the RT-LAMP assay with RT-PCR and immunofluorescence (IFT). Discussion. This RT-LAMP assay could be used to perform simultaneous and rapid detection of HTNV and SEOV to the clinical specimens. 
1 Title page

2 Ms. Ref. No.:

3 Title: Simultaneous rapid detection of Hantaan virus and Seoul virus using RT-LAMP in rats

4 Keywords: HTNV, SEOV, RT-LAMP, HFRS, sensitivity, specificity

5 Corresponding Author: Dr mingxiao ma.

6 Corresponding Author's Affiliation: Institute of Biological Sciences, Jinzhou Medical Universit

7 y, Jinzhou, China

8 First Author: Xin Sui

9 Order of Authors: Xin Sui, Xu Zhang (co-author), Dongliang Fei, Zhen Zhang, Mingxiao Ma.

10 Full address: No 40, section 3 Songpo Road Jinzhou Liaoning province China 121001

11 E-mail: lnjzmmx@163.com

12 Tel: $8604164673585 / 15941628738$

13 Fax: 8604164673528 
24 Simultaneous rapid detection of Hantaan virus and Seoul virus using RT-LAMP in rats

25 Xin Sui ${ }^{1,2}{ }^{\#}$, Xu Zhang ${ }^{1,3}{ }^{\#}$, Dongliang Fei ${ }^{1}$, Zhen Zhang ${ }^{3}$, Mingxiao Ma ${ }^{1}$ *

26 1. Institute of Biological Sciences, Jinzhou Medical University, Jinzhou, China.

27 2. The First Affiliated Hospital, Jinzhou Medical University, Jinzhou, China.

28 3. Center for Disease Control and Prevention of Jinzhou, Liaoning, China. Institute of

29 \# These authors contributed equally to this study.

$30 *$ Corresponding author:

31 Mingxiao Ma

32 Full address: No 40, section 3 Songpo Road Jinzhou Liaoning province China 121001

33 Tel: $+8604164673585 / 15941628738$

34 Fax: +8604164673528

35 E-mail: lnjzmmx@163.com

\section{ABSTRACT}

37 Background. Hemorrhagic fever with renal syndrome (HFRS) is in most cases caused by the Hantaan virus (HTNV) and Seoul virus (SEOV). To develop and apply Reverse Transcription

39 Loop-mediated Isothermal Amplification (RT-LAMP) to detect Hantaan virus (HTNV) and Seoul virus (SEOV) simultaneously, which was faster, more cost effective, and easier to perform as the target gene amplified rapidly. In this article an assay based on LAMP is demonstrated, which only employs such apparatus as a water bath or a heat block.

43 Methods. A chromogenic method using the calcein $/ \mathrm{Mn}^{2+}$ complex and real-time turbidity

44 monitoring method were used to assess reaction progress of the reaction, and the specificity of

45 the RT-LAMP-based assay was assessed by detecting cDNAs/cRNAs generated from

46 Coxsackievirus A16 (CA16), Influenza virus (IV), Lymphocytic Choriomeningitis Virus 
47 (LCM), mouse poxvirus (MPV), Rotavirus (RV), Mouse hepatitis virus (MHV). In addition,

48

49

twenty-three clinical specimens were used to determine the agreement between the RT-LAMP assay with RT-PCR and immunofluorescence (IFT) method.

Results. The detection limit of RT-LAMP to HNTV and SEOV was as low as 10 copies/ $\mu \mathrm{L}$ with optimized reaction conditions, which was much more sensitive than the RT-PCR method (1001000 copies $/ \mu \mathrm{L})$. At the same time, the detection results of twenty-three clinical specimens have also illustrated the agreement between this the RT-LAMP assay with RT-PCR and immunofluorescence (IFT).

Discussion. This RT-LAMP assay could be used to perform simultaneous and rapid detection of HTNV and SEOV to the clinical specimens.

KEY WORDS: HTNV; SEOV; RT-LAMP; HFRS; detection INTRODUCTION

Hemorrhagic fever with renal syndrome (HFRS) is characterized by fever, acute kidney damage and hemorrhage in the clinical symptoms (Schmaljohn \& Dalrymple, 1983; Zou et al., 2016; Liu et al., 2007). Most cases have been reported from mountainous areas, with about 200,000 deaths worldwide annually (Jonsson et al., 2010; Kim et al., 2016). China is the most important endemic regions of HFRS, which reach to $90 \%$ of total cases worldwide (Zou et al., 2016; Li et al., 2016). At present, HFRS is regarded as one of top nine communicable diseases in mainland China (Zou et al., 2016). At the same time, HFRS has been detected in all 31 provinces of China (Zou et al., 2016). Research have shown that HFRS is caused by hantaviruses (HVs) within the Bunyaviridae family, belonging to negative sense RNA viruses (Kim et al., 2016). In Asia and Europe, four main sero/geno types of HFRS-associated hantaviruses have been identified, including Seoul virus (SEOV), , Hantaan virus (HTNY), Dobrava-Belgrade virus (DBSV), and 
70 Puumala virus (PUUV), and among these, HTNV and SEOV are the major pathogen of HFRS in

71 China (Kim JA et al., 2016; Zhang S et al., 2014; Zhang YZ et al., 2009). HTNV RNA genome

72 contains three gene segments: L (large), M (medium), and S (small), which encode different

73 proteins, including RNA-dependent RNA polymerase (L), glycoproteins (Gn and Gc), and

74 nucleocapsid protein (N) (Kim et al., 2016). Due to serious threat to public health, it is

75 exceedingly useful that an accurate and efficient detection method of HTNV was establishment,

76 which would make more opportunity for the patients to receive treatment

77 in the early stages of HFRS disease.

78 Currently, the traditional diagnosis methods to HFRS are mainly depended on exposure history, typical clinical symptoms, IFT and serological experiment, such as IgM or IgG antibody level 80 against HTNV by enzyme-linked immunosorbent assay (ELISA). However, the traditional serological methods cann't assess the replication of the virus in patient's blood, and there is some cross-reactivity in different hantaviruses present (Lundkvist A et al., 1997; Vaheri A et al., 2008). Molecular diagnostic methods, such as the reverse transcriptase polymerase chain reaction (RT-

84 PCR) and

85 quantitative RT-PCR (qRT-PCR) assays, have been established in hantavirus detection (Jiang W

86 et al., 2014). However, these PCR-based assays require expensive instruments, specialized

87 technicians, and complicated procedures, so it is unsuitable for rapid diagnostics in field 88 situations.

89 The loop-mediated isothermal amplification (LAMP) assay is a novel method by the rapid, high

90 sensitivity, and isothermal nucleic acid amplification, which relies on Bst DNA polymerase in

91 conjunction with two inner primers and two outer primers (which recognize 6 specific areas of

92 the target gene) to amplify target gene at 
93

94

95

96

97

98

99

100

101

102

103

104

105

106

107

108

109

110

111

112

113

114

115

60-65 ${ }^{\circ}$ within $60 \mathrm{~min}$ (Notomi T et al., 2000). Since the establishment of LAMP assay in 2000, this method has been widely applied to the detection of various pathogens (Mori Y, Notomi T et al., 2009; Seki M et al., 2018; Guo XG et al., 2018; Zheng S et al., 2018), and the LAMP assay have also been used as the rapid diagnosis method of HFRS based on blood specimens of the patients (Hu et al., 2015), but blood collection isn't convenient to the rodents in the wild, and it is necessary to develop the new rapid diagnosis method of HFRS that fit for detecting the rodents in the field.

This paper reports on the establishment of a rapid detection method for HTNV and SEOV by RT-LAMP, and the S segment sequences of HTNV and SEOV were used as a target gene to be amplified in vitro transcription. This reverse transcription LAMP (RT-LAMP) assay of HTNV and SEOV has been shown to be more rapid, efficient and timesaving with compared to the traditional serological methods. Moreover, the RT-LAMP assay has similar specificity and sensitivity with RT-PCR and qRT-PCR assays, and this technique provides a novel and effective diagnostic method for HTNV and SEOV.

\section{MATERIAL \& METHODS}

\section{Ethics Statement}

This research was approved by the Experimental Animal Ethics Committee of Jinzhou Medical University (Approval: 20150918)

\section{Samples}

The purified HTNV strain RH153 and SEOV strain RH164 were gifts from Professor Liu Xuesheng of Liaoning Province Center for Disease Control and Prevention (CDC), and stored at $-80{ }^{\circ} \mathrm{C}$; Lymphocytic Choriomeningitis Virus (LCMV), mouse poxvirus (MPV), Rotavirus (RV), and Mouse hepatitis virus (MHV)-infected rat lungs were donated by Professor Zhengming He 
116 of the National Institutes for Food and Drug Control (China), and influenza virus (IV),

117 Coxsackievirus A16 (CA16), and lungs of healthy rat were preserved in Jinzhou (CDC), and

118 stored in a liquid nitrogen; The clinical lung sample were collected from the captured rat in the

119 Heishan, Guta, Beizhen and Taihe of Jinzhou during 2008-2013, which were trapped with rat

120 traps or baited cages in the tussocks, canals and fields, then stored in a liquid nitrogen until

121 further processing.

\section{Primers design}

123 By comparison with HTNV and SEOV stains of China, S gene of SEOV strain Rn-HD164

124 (GenBank: GQ279392.1) was used as the reference sequence to design the primers of RT-LAMP

125 (Fig. S1, Fig. S2 and Word S1). In order to ensure the specificity of the assay, six primers were 126 designed to identify six regions of the $\mathrm{S}$ target gene, and 5 set RT-LAMP primers for HTNV and

127 SEOV were designed based on the S gene sequence of Rn-HD164 strain using the website 128 (http://primerexplorer.jp/e/).

129 Each set of primers contained two inner primers (FIP and BIP), two outer primers (F3 and B3), 130 and two loop primers (LB and LF). The primer sequences used in the RT-PCR assays have been 131 referred to National Health and Family Planning Commission of the People's Republic of China. 132 Primers were synthesized by Shanghai Sangon Biotech, and the sequences and positions of the 133 RT-LAMP and RT-PCR primers are shown in Table 1.

\section{RNA extraction}

135 Lung tissue (about $4 \mathrm{~mm}^{3}$ ) from sample rats were obstained antiseptically in the Class II 136 biological safety cabinet, then total RNA was extracted using the Qiagen RNAmini Kit (Qiagen) 137 according to the manufacturer's directions. cDNA was synthesized with Oligo dT-Adaptor 
138 Primer and first-strand cDNA synthesis kit (Invitrogen). The contents and volume of reaction

139 mixture were as follows: $\mathrm{MgCl}_{2} 2 \mu \mathrm{L}, 10 \times \mathrm{RT}$ Buffer $1 \mu \mathrm{L}$, RNase Free $\mathrm{dH}_{2} \mathrm{O} 3.75 \mu \mathrm{L}$, dNTP

140 Mixture $(10 \mathrm{mM}) 1 \mu \mathrm{L}$, RNase Inhibitor $0.25 \mu \mathrm{L}$, AMV Reverse Transcriptase $0.5 \mu \mathrm{L}$, Oligo dT-

141 Adaptor Primer $0.5 \mu \mathrm{L}$ which made up to $10 \mu \mathrm{L}$. The reaction conditions were as follows: $30^{\circ} \mathrm{C}$

142 for $10 \mathrm{~min}, 42^{\circ} \mathrm{C}$ for $30 \mathrm{~min}, 50^{\circ} \mathrm{C}$ for $30 \mathrm{~min}, 95^{\circ} \mathrm{C}$ for $15 \mathrm{~min}$, and $5^{\circ} \mathrm{C}$ for $5 \mathrm{~min}$. The cDNA

143 was stored at $-20^{\circ} \mathrm{C}$. Viral RNA was extracted using the TIANamp Virus DNA/RNA

144 Kit (Tiangen, Beijing, China) according to the manufacturer's instructions.

145 RT-LAMP assays

146 The LAMP reaction was performed in $25-\mu \mathrm{L}$ volume which contained $20 \mathrm{mM}$ Tris- $\mathrm{HCl}(\mathrm{pH} 8.8)$

147 (Shanghai Bioscience Biological Technology Company), $10 \mathrm{mM} \mathrm{KCl}$ and $10 \mathrm{mM}\left(\mathrm{NH}_{4}\right)_{2} \mathrm{SO}_{4}$

148 (Sinopharm Chemical Reagent Co.), 0.1\% Triton X-100 (Mylab Corporation), 0.8 M lysine

149 (Sigma-Aldrich), 8 mM MgSO 4 (Sinopharm Chemical Reagent Co.), 1.4 mM dNTP, 8 U of Bst

150 DNA polymerase and reverse transcriptase, 40 pmol of each primer FIP and BIP, 5 pmol of each

151 primer $\mathrm{F} 3$ and $\mathrm{B} 3$, and 20 pmol of each primer $\mathrm{LB}$ and $\mathrm{LF}$. The reaction was performed at $65^{\circ} \mathrm{C}$

152 for $60 \mathrm{~min}$, and double-distilled water was used as a negative control.

153 LAMP products were analyzed by two independent methods, either sample turbidity or 154 fluorescence. The LA-320 turbidimeter (Eiken Chemical Co., Ltd) was used to monitor the 155 turbidity of RT-LAMP products in real time (measured at 6-s intervals on $650 \mathrm{~nm}$ wave length), 156 and the samples were considered positive if the value of sample turbidity exceeded 0.1 . The 157 turbidity changes arose from the presence of LAMP reaction by-product a white precipitate $158\left(\mathrm{Mg}_{2} \mathrm{P}_{2} \mathrm{O}_{7}\right)$. The second method used visual inspection to assess color changes in the presence of 159 the fluorescent metal ion indicator calcein $/ \mathrm{Mn}^{2+}$ complex, and the samples that turned from 
160 orange to green on reaction completion, while those, no color change samples, were considered 161 negative.

162

\section{Optimization of primers and temperature for RT-LAMP assay}

To determine the optimal primer for RT-LAMP assay for HTNV and SEOV detection, five primer sets were detected in the same reaction condition using real-time turbidimeter LA-320 and their turbidity curves were draw at $650 \mathrm{~nm}$ according to the amplified results. Then the optimal primers were used to initiate RT-LAMP reaction at different temperatures ranging from $59^{\circ} \mathrm{C}$ to $66^{\circ} \mathrm{C}$ with a gradient of $1^{\circ} \mathrm{C}$.

\section{RT-PCR assays}

RT-PCR reaction was conducted in accordance with the manufacturer's protocol. Briefly, $5 \mu \mathrm{L}$ of RNA sample was added to a $20 \mu \mathrm{L}$ reaction mixture containing $100 \mathrm{ng}$ Oligo dT (15mer), $1 \mathrm{X}$ AMV/Tfl buffer buffer , $1 \mathrm{mmol} / 1 \mathrm{MgSO} 4,10 \mathrm{mmol} / 1 \mathrm{DTT}, 200 \mu \mathrm{mol} / 1$ of each dNTP, 300nM each specific primer, 2.5 units each of AMV Reverse Transcriptase and Tfl DNA Polymerase and $20 \mathrm{U}$ RNasin. RT-PCR assays was followed uninterrupted thermal cycling programs consisting of 45 minutes at $42^{\circ} \mathrm{C}, 4$ minutes at $94^{\circ} \mathrm{C}, 35$ cycles of 30 seconds at $94^{\circ} \mathrm{C}, 30$ seconds at $55^{\circ} \mathrm{C}$ and 30 seconds at $72^{\circ} \mathrm{C}$ and a final elongation step of 10 minutes at $72^{\circ} \mathrm{C}$. RTPCR products were electrophoresed in 1\% agarose gel and visualized using UV light after ethidium bromide staining.

\section{Sensitivity comparison of RT-LAMP and RT-PCR}

For comparison of sensitivity between RT-LAMP assay and RT-PCR, the positive controls (cRNA templates of the purified HTNV strain RH153 and SEOV strain RH164) were detected on the same samples. After the cRNA template concentration of the purified HTNV and SEOV was measured using the NanoDrop One microvolume UV-Vis spectrophotometer 
183 (ThermoFisher, Waltham, USA), the viral copy numbers were calculated from the full length 184 genome using the formula (copies $/ \mu \mathrm{L}=\mathrm{ng} / \mu \mathrm{L} \times$ Avogadro constant $/ 1$ bases of average molecular 185 mass $\times$ genome length), then seven assay standards were prepared by 10-fold serial dilutions 186 containing $10^{5}-10^{-1}$ copies/ $\mu \mathrm{L}$ respectively. the cRNA of the healthy rat lung tissue was used as

187 the negative control. The colorimetric method (the addition of calcein) and the turbidity method 188 (determination of turbidity) were both used to monitor the reaction of LAMP. At the same time, 189 the RT-PCR products were electrophoresed in 1\% agarose gel and visualized using UV light.

190 Specificity test of RT-LAMP

191 The specificity of RT-LAMP was assessed by testing cDNAs generated from Coxsackievirus 192 A16 (CA16), Influenza virus (IV), Lymphocytic Choriomeningitis Virus (LCM), mouse 193 poxvirus (MPV), Rotavirus (RV), Mouse hepatitis virus (MHV), lungs of healthy rats and HTNV,

194 SEOV positive samples. Distilled water was used as negative control.

195 Examination of clinical samples

196 Twenty-three rat lung samples were randomly selected from those collected between 2008 and

197 2013. All samples were collected according to the national guideline for prevention and

198 treatment of epidemic hemorrhagic fever which states that night trapping method was used to

199 seize rats randomly in the countryside and residential area(Zhang YZ et al., 2004). Cordotomy

200 was used to kill all the rats. Each of them was tested with RT-PCR, IFT and RT-LAMP.

201 RESULTS

202 Optimization of primers

203 To determine the optimal primers for HTNV and SEOV, five primers were detected in the same

204 reaction condition using real-time turbidimeter L320C and their turbidity curves were screened at $205650 \mathrm{~nm}$ according to the amplified results. As illustrated in Fig. 1, HS-72 was selected as optimal 206 primers (Table 1). 
208 The optimal primers HS-72 were used to initiate RT-LAMP reaction at different temperatures

209 ranging from $59^{\circ} \mathrm{C}$ to $66^{\circ} \mathrm{C}$ with a gradient of $1{ }^{\circ} \mathrm{C}$. As shown in Fig. 4, RT-LAMP was first

210 initiated at $64^{\circ} \mathrm{C}, 65^{\circ} \mathrm{C}$, and $66^{\circ} \mathrm{C}$. However, the higher temprature could have an effect on the

211 activity of Bst DNA polymerase and the reaction time was longer with temperature increasing,

212 so $65^{\circ} \mathrm{C}$ was selected as the optimal temperature.

213 Specificity test

214 HS-72 was used with all the provided samples in subsequent reactions. HS-72 displayed high

215 specificity and could be used for simultaneous detection of HTNV and SEOV. Only HTNV and

216 SEOV were detected with HS-72 and none of the samples with other viruses were diagnosed as

217 positive, suggesting a good specificity (Fig. 2A-B).

218 Sensitivity test and comparison with PCR

219 For templates at concentrations from $1 \times 10^{5}$ to $1 \times 10^{-1}$ copies/L, RT-LAMP assays were carried 220 out by the LA-320C turbidimeter. As illustrated in Fig. 3A-D, both the turbidity and color 221 detection limit of the RT-LAMP assay was up 10 copies/ $\mu 1$ for HTNV and SEOV. Comparison 222 to the the RT-LAMP assay, the RT-PCR limits of detection for HTNV and SEOV were $10^{2}$ and $22310^{3}$ copies/L, respectively (Fig. 3E-F).

\section{Test results of clinical samples}

225 The 23 clinical samples were detected to determine the feasibility of the RT-LAMP assay, and 226 result have shown in Fig. 5A and B. At the same time, all the samples were subjected to 227 conventional RT-PCR and IFT. In the result, the RT-LAMP method showed that five samples 228 were determined as the positive of SEOV and HTNV, and four positive specimen in RT-PCR 229 and only three in the IFT assay, which was superior to RT-PCR and IFT methods (Tab.2). From 230 shown in Table 2, all three methods yielded positive results for the JZHS-5, LZLH-4, JZGT-1 
231 samples, and and JZGT-2 sample wasn't detected using RT-PCR, but the detection of RT-LAMP

232 were positive.

\section{DISCUSSION}

234 Since its development, LAMP has been widely applied in diagnosis of clinical illness, 235 quantitative and qualitative detection of epidemic bacteria and viruses, and gender determination 236 of animal embryos (Mori Y, Notomi T et al., 2009; Seki M et al., 2018; Guo XG et al., 2018;

237 Zheng S et al., 2018; Hirayama $\mathrm{H}$ et al., 2006). Hantaviruses are among the most important 238 zoonotic pathogens of humans and the subject of heightened global attention (Guo et al., 2013), 239 and previous studies show that rodents have long been recognized as the principal reservoirs of 240 hantaviruses (Myriam et al., 2016), so it is important to develop effectively measures to 241 investigate HFRS infection in rodents in the wild. Although LAMP have been used as the rapid 242 diagnosis method of HFRS based on blood specimens of the patients (Hu et al., 2015), but the 243 sensitivity for HTNV was 10-fold lower than that for SEOV in this assay which probably led to 244 undetection for HTNV, and the blood collection isn't convenient to the rodents in the wild, so the method was unsuitable to rapid epidemiological investigation of hantaviruses (Myriam et al., 2016). In this research, specific primers for HTNV and SEOV were designed to carry out RTLAMP using lung tissue specimens, and the new method is more suitable to apply in the wild. At the same time, the new RT-LAMP assay not only showed high sensitivity and rapidity, but also could be finished in less than $1 \mathrm{~h}$ with only a heated water bath required, so this method appears to be more suitable in the endemic areas with poorly equipped laboratories. Currently, the detection cost to each sample of RT-LAMP assay is about three times as compared to RT-PCR method, but the cost will be remarkably reduced as the extensive application. Furthermore, most researchers recommend electrophoresis of amplification products or addition of SYBR Green I 
254 fluorochrome to the products as a monitoring method. However, both these methods expose the

255 products to the atmosphere, thus increasing the risk of false positive results. Additionally,

256 traditional electrophoresis methods fail to perform real-time monitoring. Our study employs real-

257 time nephelometry and spectrophotometry without opening the reaction tube. Therefore,

258 contamination is avoided, thereby preventing false positive outcomes.

259 RT-LAMP showed better potential than PCR in many aspects. Significantly, RT-LAMP featured

260 isothermal amplification, which is independent of the thermal cycler system, thus promoting

261 application of LAMP. It is known that addition of lysine facilitates isothermal amplification in

262 presence of Bst DNA polymerase by achieving dynamic equilibrium between DNA denaturation

263 and renaturation. Further, Notomi found that the existence of impurities and huge amounts of

264 exogenous DNA hardly affected the RT-LAMP reaction (Notomi T, 2007). It has been shown

265 that 6 copies of HBV DNA could be detected by the LAMP reaction without being interfered

266 with by $100 \mathrm{ng}$ of human DNA. In a study by Kaneko, extraction of DNA from the sample was

267 required due to the insusceptibility of LAMP to other components in the samples (Kaneko H et

268 al., 2007). However, PCR can be disturbed by the presence of exogenous DNA and inhibitors in

269 the samples. Hence, LAMP is more suitable for clinical samples than PCR. In addition, the

270 specificity of the LAMP reaction was much higher on account of its specific recognition of six

271 distinct regions by four primer pairs (Notomi T et al., 2000). In contrast, only two distinct

272 regions are recognized in PCR. Therefore, the false positive rate of LAMP was largely lowered.

273 In our study, a combination of optimal primer pair HS-72 was selected. The optimal

274 concentration of each set of primers was as follows: 40 pmol FIP and BIP, 5 pmol F3 and B3, 20

275 pmol LB and LF. The optimal reaction temperature was $65^{\circ} \mathrm{C}$. The optimal reaction time was 60

276 minutes. 
277 In this study, RT-LAMP was used for the rapid and simultaneous detection of HTNV and SEOV.

278 The specificity test demonstrated that the target genes of HTNV and SEOV could be specifically

279 amplified. The sensitivity detection demonstrated a detection limit of 10 copies/ $\mu 1$ of HTNV and

280 SEOV, and this assay is superior to traditional RT-PCR and IFT methods. In summary, RT-

281 LAMP is suited for sensitive and rapid detection of HTNV and SEOV. Because of its simplicity

282 and efficiency, this method is likely to be used to detect HTNV and SEOV in samples collected

283 in the field. This will enable researchers to conduct epidemiological investigations that could

284 provide the basis for prevention of HTNV and SEOV.

\section{CONCLUSIONS}

286 The method for the detection of both HTNV and SEOV has been developed in this study, which

287 is of great significance for the diagnosis of HFRS. With better sensitivity and comparable 288 specificity to current diagnostic tests, and better availability in laboratories of various standards, 289 this method has the potential to be utilized on a large scale.

\section{ETHICS STATEMENT}

291 The animal samples used in this research were collected according to the National Scheme for 292 the prevention and Treatment of epidemic hemorrhagic fever. The trappers were permitted to 293 trap the rats according to the National Scheme for Supervision of HFRS which was a multi-level 294 scheme from country to city. This research was approved by the Experimental Animal Ethics 295 Committee of Jinzhou Medical University.

\section{COMPETING INTERESTS}

297 The authors declare that they have no competing interests.

\section{AUTHORS' CONTRIBUTIONS}


299 Mingxiao Ma designed the overall research; Xu Zhang and Dongliang Fei were responsible for

300 the design of primers, specificity test, and sensitivity test; Xin Sui and Zhen Zhang were

301 responsible for the collection of specimens. All authors read and approved the final manuscript.

302 ACKNOWLEDGEMENTS

303 This research was awarded by "Lioning Distinguished Professor".

304 REFERENCES

305 Guo XG, Zhou YZ, Li Q, Wang W, Wen JZ, Zheng L, Wang Q. 2018.Rapid and reliable

306 diagnostic method to detect Zika virus by real-time fluorescence reverse transcription loop-

307

308

309

310

311

312

313

314

315

316

317

318

319

320

321

Guo WP, Lin XD, Wang W, Tian JH, Cong MC, Zhang HL, Wang MR, Zhou RH, Wang

JB, Li JG, Holmes EC, Zhang YZ. 2013. Phylogeny and Origins of Hantaviruses

Harbored by Bats, Insectivores, and Rodents. PLoS Pathog, 9(2): e1003159.

Hirayama H, Kageyama S, Takahashi Y, Moriyasu S, Sawai K, Onoe S, Watanabe K, Kojiya S, Notomi T, Minamihashi A. 2006.Rapid sexing of water buffalo (Bubalus bubalis) embryos using loop-mediated isothermal amplification. Theriogenology, $66(5), 1249-56$.

Hu D, Hao L, Zhang J, Yao P, Zhang Q, Lv H, Gong X, Pan X, Cao M, Zhu J, Zhang Y, Feng Y, Wang C. 2015.Development of reverse transcription loop-mediated isothermal amplification assays to detect Hantaan virus and Seoul virus. J Virol Methods, 221: 68-73.

Jiang W, Wang PZ, Yu HT. 2014. Development of a SYBR Green I based one-step real-time PCR assayfor the detection of Hantaan virus. Journal of Virological Methods, 196,145-151.

Jonsson CB, Figueiredo LT, VapalahtiO. 2010 .A global perspective on han-tavirus ecology, epidemiology, and disease. Clinical Microbiology Reviews, 23, 412-441. 
322 Kaneko H1, Kawana T, Fukushima E, Suzutani T. 2007. Tolerance of loop-mediated

323 isothermal amplification to a culture medium and biological substances. J Biochem Biophys 324 Methods, 70(3), 499-501.

325 Kim JA, Kim WK, No JS, Lee SH, Lee SY, Kim JH, Kho JH, Lee D, Song DH, Gu

326 SH, Jeong ST, Park MS, Kim HC, Klein TA, Song JW. 2016.Genetic Diversity and

327 Reassortment of Hantaan Virus Tripartite RNA Genomes in Nature, the Republic of Korea. $328 \quad$ PLoS Negl Trop Dis, 10(6), e0004650.

329

330

331

332

333

334

335

336

337

338

339

340

341

342

343

Kim WK, Kim JA, Song DH, Lee D, Ki m YC, Lee SY, Lee SH, No JS, Kim JH, Kho JH, Gu SH, Jeong ST, Wiley M, Kim HC, Klein TA, Palacios G, Song JW. 2016. Phylogeographic analysis of hemorrhagic fever with renal syndrome patients using multiplex PCR-based nextgeneration sequencing. Scientific Reports, 6: 26017. doi: 10.1038/srep26017.

Li Q, Cai YA, Wei YM, Han X, Han ZY, Zhang YB, Qi SX, Xu YG.2016.Genovariation Study of Hantavirus in Main Endemic Areas of Hemorrhagic Fever with Renal Syndrome in Hebei Province China. PLOS ONE, 11(7), e0159731.

Liu YX, Feng D, Zhang Q, Jia N, Zhao ZT, De Vlas SJ.2007. Key differentiating features between scrub typhus and hemorrhagic fever with renal syndrome in northern China. Am J Trop Med Hyg, 76, 801-805.

Lundkvist A, Hukic M, Horling J, Gilljam M, Nichol S, Niklasson B. 1997. Puumala and Dobrava viruses cause hemorrhagic fever with renal syndrome inBosnia-Herzegovina: evidence of highly cross-neutralizing antibody responsesin early patient sera. J Med Virol, $53,51-59$. 
344 Mori Y, Notomi T. 2009. Loop-mediated isothermal amplification (LAMP): a rapid, accurate,

345 and cost-effective diagnostic method for infectious diseases. Journal of infection and

$346 \quad$ chemotherapy, 15(2), 62-69.

347 Myriam Ermonval, Florence Baychelier and Noël Tordo. 2016. What Do We Know about

348 How Hantaviruses Interact with Their Different Hosts. Viruses, 2016, 8(8): 223-240.

349 Notomi T, Okayama H, Masubuchi H, Yonekawa T, Watanabe K, Amino N, Hase T. 2000.

350 Loop-mediated isothermal amplification of DNA. Nucleic Acids Res, 28, e63-e63.

351

352

353

354

355

356

357

358

359

360

361

362

363

364

365

Notomi T, Okayama H, Masubuchi H, Yonekawa T, Watanabe K, Amino N, Hase T .2000. Loop-mediated isothermal amplification of DNA. Nucleic acids research, 28(12), e63-e63

Notomi T. 2007. Loop-mediated isothermal amplification. Nihon rinsho. Japanese journal of clinical medicine, 65(5), 957-961

Schmaljohn CS, Dalrymple JM. 1983. Analysis of Hantaan virus RNA: evidence for a new genus of bunyaviridae. Virology, 131, 482-491.

Seki M, Kilgore PE, Kim EJ, Ohnishi M, Hayakawa S, Kim DW. 2018 .Loop-Mediated Isothermal Amplification Methods for Diagnosis of Bacterial Meningitis. Front Pediatr, 6:57. Doi, 10.3389/fped.2018.00057. eCollection .

Vaheri A, Vapalahti O, Plyusnin A. 2008. How to diagnose hantavirus infectionsand detect them in rodents and insectivores. Rev Med Virol, 18, 277-288.

Zhang S, Wang S, Yin W, Liang M, Li J, Zhang Q, Feng Z, Li D. 2014. Epidemic characteristics of hemorrhagic fever with renal syndrome in China, 2006-2012 . BMC Infect Dis, 14,384 . 
366 Zhang YZ, Xiao DL, Wang Y, Wang HX, Sun L, Tao X X, Qu YG . 2004. The epidemic

367 characteristics and preventive measures of hemorrhagic fever with syndromes in China.

368 Zhonghua liu xing bing xue za zhi, 25(6), 466-469

369 Zhang YZ, Zhang FX, Wang JB, Zhao ZW, Li MH, Chen HX, Zou Y, Plyusnin A. 2009.

370 Hantaviruses in rodents and humans, Inner Mongolia Autonomous Region, China. Emerg.

371 Infect Dis, 15, 885-891.

372

373

374

375

376

377

378

379

380

381

382

383

384

385

386

387

388

\section{Zheng S, Wu X, Shi J, Peng Z, Gao M, Xin C, Liu Y, Wang, Xu S, Han H, Yu J, Sun W,} Cong X, Li J, Wang J. 2018. Rapid specific and visible detection of porcine circovirus type 3 using loop-mediated isothermal amplification (LAMP). Transbound Emerg Dis. Doi, $10.1111 /$ tbed.12835.

Zou LX, Chen MJ, Sun L. 2016. Haemorrhagic fever with renal syndrome: literature review and distribution analysis in China. Int J Infect Dis., 43(2),95-100. 
390 Figure Legends

391 Fig. 1 The results of RT-LAMP with different sets of primers.

392 Each set of primers was tested with the same concentration of templates. Turbidity indicated the

393 level of amplification. The reaction mixture with HS-72 achieved a turbidity of approximate 0.06

394 in $15 \mathrm{~min}$, while others were below 0.017 at this point.

395

396 Fig. 2 The specificity assay of RT-LAMP.

397 A Results were analyzed using an LA-320C turbidimeter: Amplification was carried out with 398 the same reaction mixture and the same concentration of different templates, and only the 399 reaction mixture with HTNV and SEOV samples showed an amplification curve, and no 400 amplification was found with other samples.

401 B Results were analyzed through color changes: Tube 1, Lung of healthy rat; 2, Negative 402 control; 3, CA16; 4, LCMV; 5, HTNV; 6, IV; 7, MPV; 8, MHV; 9, RV; 10, SEOV.

403

404 Fig. 3 Optimal temperature assay of RT-LAMP.

405 Turbidity indicated the level of amplification. The reaction at $65^{\circ} \mathrm{Cshowed}$ a turbidity of 0.044 at 406 as early as $12 \mathrm{~min}$, while the turbidity of other reactions was below 0.001 at this time.

407

408 Fig. 4 Comparison of hantavirus detection sensitivity by RT-LAMP and RT-PCR assays.

409 Results were analyzed using an LA-320C turbidimeter (A and B): both HTNV and SEOV were

410 successfully detected with a detection range of 10-100000 copies/ $\mu 1$, but the samples with a

411 concentration of 1 copy $/ \mu 1$ and 0.1 copy/ $\mu 1$ wasn't detected, and

412 the negative control showed no amplification either.

413 Color formation indicated the level of amplification (C and D). From right to left: $10^{5}$ copies $/ \mu 1$,

$41410^{4}$ copies $/ \mu 1,10^{3}$ copies $/ \mu 1,10^{2}$ copies $/ \mu 1,10^{1}$ copies $/ \mu 1,10^{0}$ copies $/ \mu 1,10^{-1}$ copies $/ \mu 1$, and

415 negative control. A significant difference in reaction color was observed in presence of

416 amplification (the left five green tubes) and in absence of amplification (the three yellow ones on 417 the right). 
418 Results of the sensitivity assay using RT-PCR: From right to left: $10^{5}$ copies $/ \mu 1,10^{4}$ copies $/ \mu 1$, $41910^{3}$ copies $/ \mu 1,10^{2}$ copies $/ \mu 1,10^{1}$ copies $/ \mu 1,1$ copies $/ \mu 1,10^{-1}$ copies $/ \mu 1$, negative control. DNA 420 marker: D2000. HTNV and SEOV could be detected at as low as 100-1000 copies/ $\mu 1$.

421

422 Fig.5 Detection of clinical specimens by RT-LAMP assay.

423 A: Results were analyzed using the LA-320C turbidimeter: JZGT-2, JZTH-5, JZGT-1, JZLH-4 424 and JZHS-5 were determined to be positive in the 23 clinical specimens.

425 B: Results were analyzed through color changes:

426 1-8: JZHS-1, JZHS-2, JZGT-2, JZLH-1, JZLH-2, JZLH-3, JZTH-5, JZHS-3;

427 2-16: JZHS-4, JZGT-3, JZGT-4, JZGT-1, JZBZ-1, JZBZ-2, JZBZ-3, JZBZ-4;

428 17-23: JZLH-4, JZBZ-5, JZTH-1, JZHS-5, JZTH-2, JZTH-3, JZTH-4; 


\section{Table 1 (on next page)}

Tab.1 The primers used for RT-LAMP and RT-PCR.

Tab.1 The primers used for RT-LAMP and RT-PCR. 
Tab.1 The primers used for RT-LAMP and RT-PCR.

\begin{tabular}{|c|c|c|c|c|}
\hline Set & Reaction Type & Primer Number & Position & Sequence(5'-3') \\
\hline \multirow[t]{6}{*}{ I } & RT-LAMP & HS-72F3 & $388-405$ & ACAGCTGATTGGTTGACT \\
\hline & & HS-72B3 & $584-601$ & TTGATTGGGCATTTGGCA \\
\hline & & HS-72FIP & $406-430,453-477$ & ACCTCTTGTTGTTAACATGTACAGTTTATAATTGTCTATCTGACATCATTCG \\
\hline & & HS-72BIP & $509-531,563-583$ & GGATCAGATTCAAGGATGACAGCTTTTGACACATACAGATGTTTGG \\
\hline & & HS-72LF & & GCCTTCAAGATGATTGGGACCA \\
\hline & & HS-72LB & & TGAGGATGTCAATGGAATCAGAAAG \\
\hline \multirow[t]{5}{*}{ II } & RT-LAMP & HS-154F3 & $647-666$ & TATGTGGGTTATATCCTGCA \\
\hline & & HS-154B3 & $826-847$ & TCTGTCTGATATAGTCACGATT \\
\hline & & HS-154FIP & $709-728,669-689$ & GCCAGTGCCAAAAACCCAACTTGATAAAGGCAAGGAACATGGT \\
\hline & & HS-154BIP & $743-766,803-822$ & CTAGAATTGAAGAATGGCTTGGCGTTAGGATTCCCAGATAAACTCC \\
\hline & & HS-154LB & & CACCCTGCAAGTTCATGGCAGAG \\
\hline \multirow[t]{5}{*}{ III } & RT-LAMP & HS-203F3 & $803-822$ & GGAGTTTATCTGGGAATCCT \\
\hline & & HS-203B3 & $976-993$ & TGGTGGACACCTATCAGG \\
\hline & & HS-203FIP & $824-848,864-886$ & GAAATTCCTTTGGCTCCATTCCTTTTAAATCGTGACTATATCAGACAGAG \\
\hline & & HS-203BIP & $945-963,892-911$ & CTCAGGCAACATGCAAAGGATTCACCCATATTGACGATGGT \\
\hline & & HS-203LB & & TGCTGGATGTACACTGGTTGA \\
\hline \multirow[t]{5}{*}{ IV } & RT-LAMP & HS-1F3 & $15-39$ & CTAAAGAGCTATTACACTAACAAGA \\
\hline & & HS-1B3 & $215-232$ & GGCGCTTCAATTCATCAA \\
\hline & & HS-1FIP & $85-104,44-64$ & GCTATCACAAGCTGCCСCTCTTTGGCAACTATGGAAGAAATCC \\
\hline & & HS-1BIP & $121-145,183-200$ & GATGCAGAAAAGCAGTATGAGAAGGTTGAAGCTGCAACACTCTCC \\
\hline & & HS-1LB & & ATCCTGATGACTTAAACAAGAGGG \\
\hline \multirow[t]{5}{*}{$\mathrm{V}$} & RT-LAMP & HS-124F3 & $539-559$ & AGGATGTCAATGGAATCAGAA \\
\hline & & HS-124B3 & $702-721$ & CСAAAAACCCAACTACACTC \\
\hline & & HS-124FIP & $601-625,561-580$ & GTGTTATCTCTTCAGCTTTCATGCTTTGCCCAAACATCTGTATGTGT \\
\hline & & HS-124BIP & $629-648,684-701$ & GAAGATTCCGCACGGCAGTATTATGACAGGGCTTACCATG \\
\hline & & HS-124LB & & TGTGGGTTATATCCTGCACAGA \\
\hline \multirow[t]{2}{*}{ VI } & RT-PCR & HHSIF & $688-709$ & GTAAGCCCTGTCATGAGTGTAG \\
\hline & & HHSIQ & $885-907$ & TTGCATGTTGCCTGAGGGCTTGA \\
\hline
\end{tabular}

2 Note: The gene region used for designing primers from the S gene (GenBank: GQ279392). 


\section{Table 2 (on next page)}

Tab.2. Summary of test results of 23 clinical specimens.

Tab.2. Summary of test results of 23 clinical specimens. 
Tab.2. Summary of test results of 23 clinical specimens.

\begin{tabular}{|c|c|c|c|c|}
\hline Samples & Area of collecting sample & RT-PCR & RT-LAMP & IFT \\
\hline JZHS-1 & Heishan & - & - & - \\
\hline JZHS -2 & Heishan & - & - & - \\
\hline JZHS -3 & Heishan & - & - & - \\
\hline JZHS -4 & Heishan & - & - & - \\
\hline JZHS -5 & Heishan & + & + & + \\
\hline JZLH-1 & Linghai & - & - & - \\
\hline JZLH-2 & Linghai & - & - & - \\
\hline JZLH -3 & Linghai & - & - & - \\
\hline JZLH -4 & Linghai & + & + & + \\
\hline JZGT-1 & Guta & + & + & + \\
\hline JZGT -2 & Guta & - & - & + \\
\hline JZGT -3 & Guta & - & - & - \\
\hline JZGT -4 & Guta & - & - & - \\
\hline JZBZ-1 & Beizhen & - & - & - \\
\hline JZBZ-2 & Beizhen & - & - & - \\
\hline JZBZ-3 & Beizhen & - & - & - \\
\hline JZBZ-4 & Beizhen & - & - & - \\
\hline JZBZ-5 & Beizhen & - & - & - \\
\hline JZTH-1 & Taihe & - & - & - \\
\hline JZTH-2 & Taihe & - & - & - \\
\hline JZTH-3 & Taihe & - & - & - \\
\hline JZTH-4 & Taihe & - & - & - \\
\hline JZTH-5 & Taihe & + & - & + \\
\hline
\end{tabular}

2

3

4

5 
Figure 1

Fig. 1 The results of RT-LAMP with different sets of primers.

Each set of primers was tested with the same concentration of templates. Turbidity indicated the level of amplification. The reaction mixture with HS-72 achieved a turbidity of approximate 0.06 in $15 \mathrm{~min}$, while others were below 0.017 at this point.

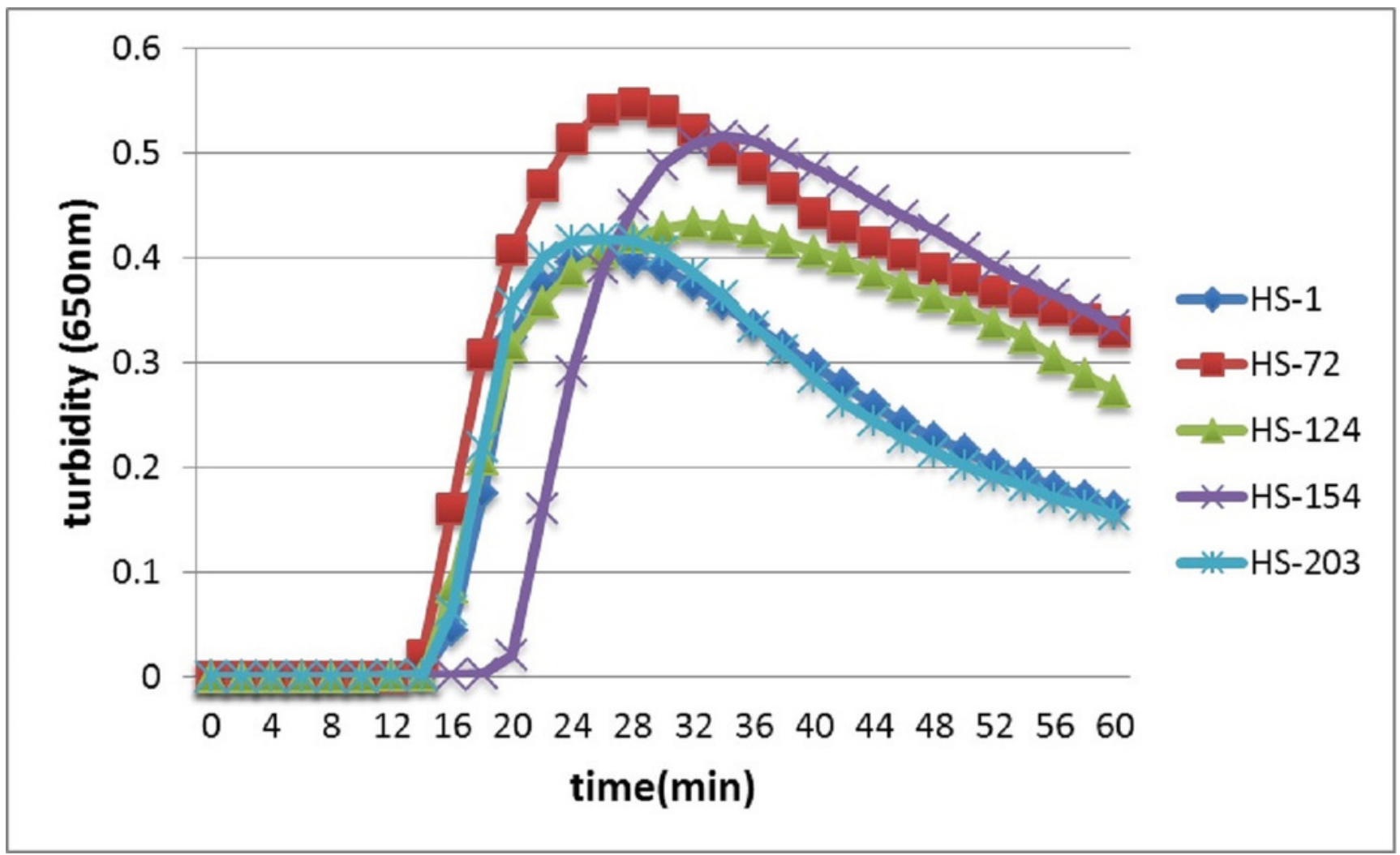




\section{Figure 2}

Fig. 2 The specificity assay of RT-LAMP.

A Results were analyzed using an LA-320C turbidimeter: Amplification was carried out with the same reaction mixture and the same concentration of different templates, and only the reaction mixture with HTNV and SEOV samples showed an amplification curve,and no amplification was found with other samples. B Results were analyzed through color changes: Tube 1, Lung of healthy rat; 2, Negative control; 3, CA16; 4, LCMV; 5, HTNV; 6, IV; 7, MPV; 8 , MHV; 9, RV; 10, SEOV. 


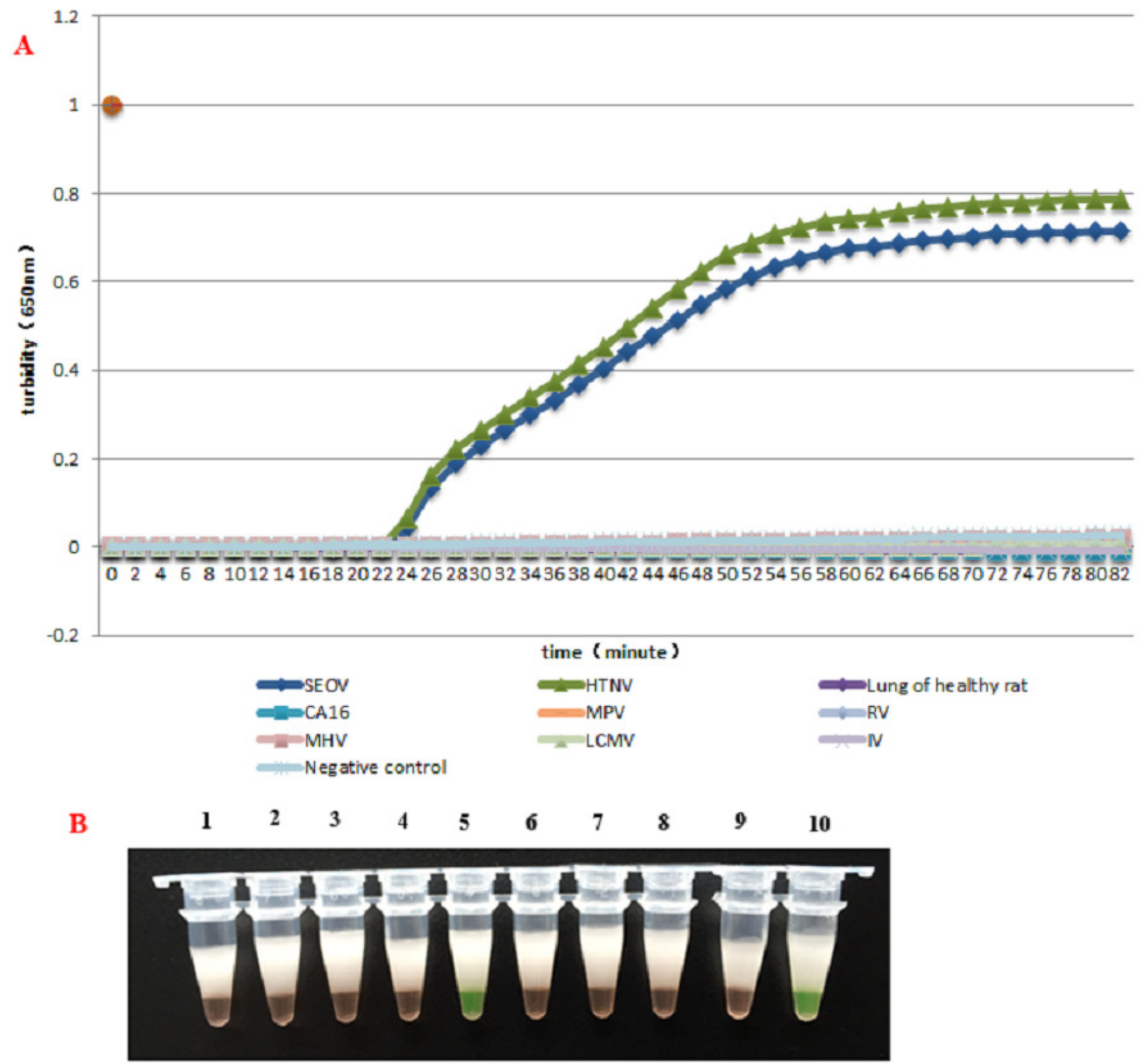


Figure 3

Fig. 3 Optimal temperature assay of RT-LAMP.

Turbidity indicated the level of amplification. The reaction at $65^{\circ} \mathrm{C}$ showed a turbidity of 0.044 at as early as $12 \mathrm{~min}$, while the turbidity of other reactions was below 0.001 at this time.

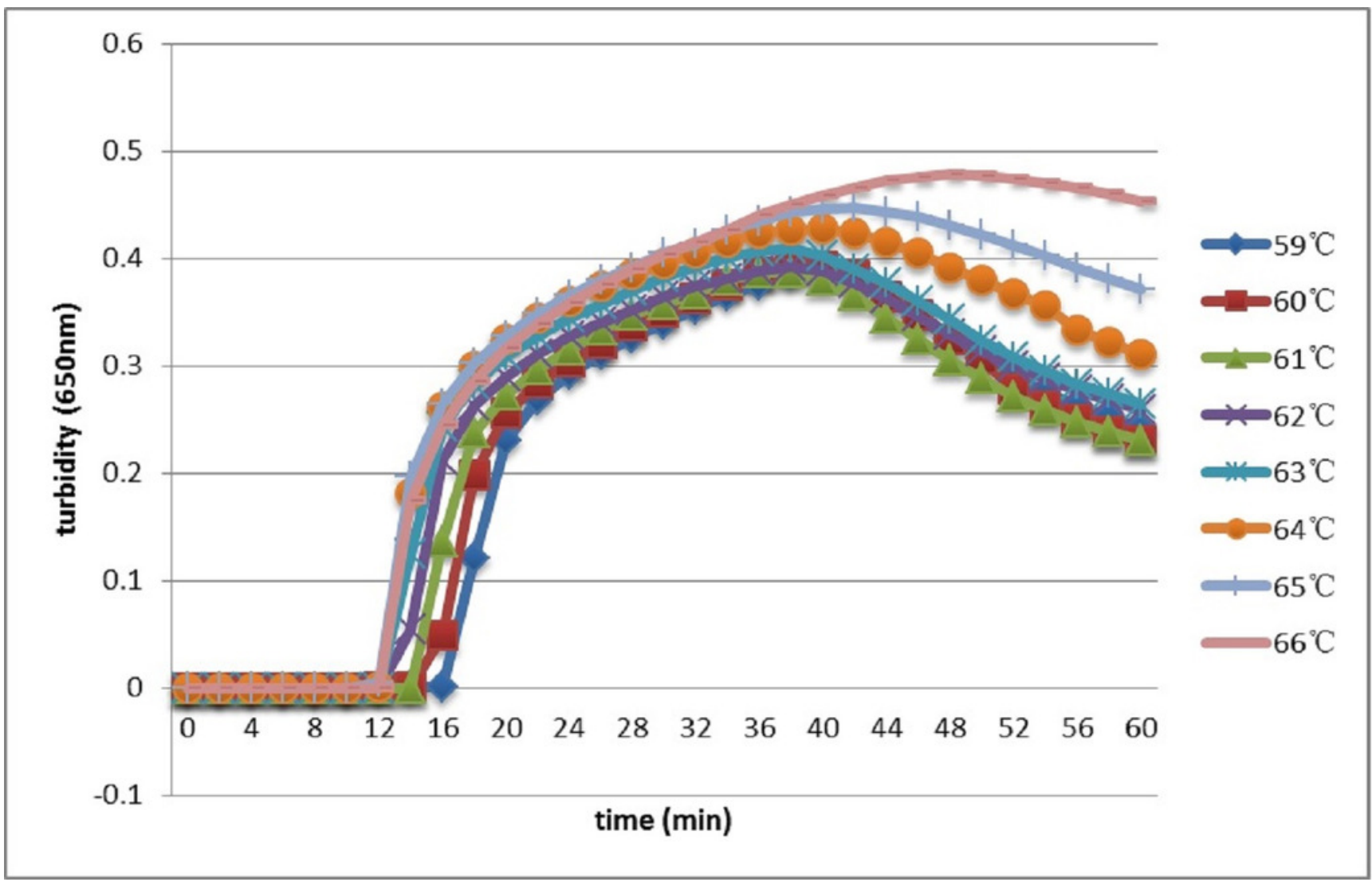




\section{Figure 4}

Fig. 4 Comparison of hantavirus detection sensitivity by RT-LAMP and RT-PCR assays.

Results were analyzed using an LA-320C turbidimeter (A and B): both HTNV and SEOV were successfully detected with a detection range of 10-100000 copies/ $\mu l$, but the samples with a

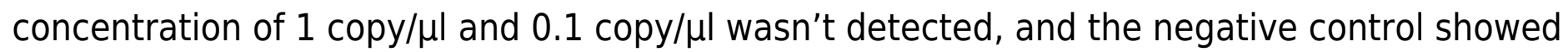
no amplification either. Color formation indicated the level of amplification ( $C$ and $D$ ). From right to left: $10^{5}$ copies $/ \mu \mathrm{l}, 10^{4}$ copies $/ \mu \mathrm{l}, 10^{3}$ copies $/ \mu \mathrm{l}, 10^{2}$ copies $/ \mu \mathrm{l}, 10^{1} \mathrm{copies} / \mu \mathrm{l}, 10^{0}$ copies $/ \mu \mathrm{l}, 10^{-1} \mathrm{copies} / \mu \mathrm{l}$, and negative control. A significant difference in reaction color was observed in presence of amplification (the left five green tubes) and in absence of amplification (the three yellow ones on the right). Results of the sensitivity assay using RTPCR: From right to left: $10^{5}$ copies $/ \mu l, 10^{4}$ copies $/ \mu l, 10^{3}$ copies $/ \mu l, 10^{2}$ copies $/ \mu \mathrm{l}, 10^{1}$

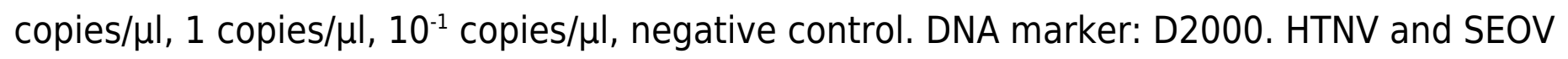
could be detected at as low as $100-1000$ copies/ $\mu$ l. 
A

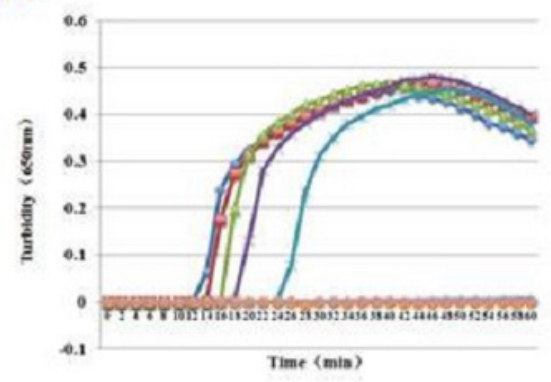

C

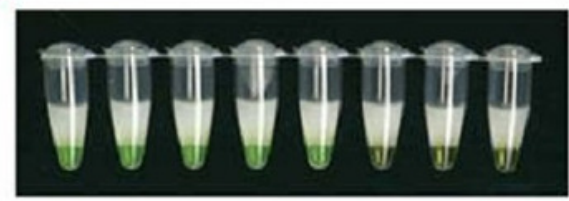

E

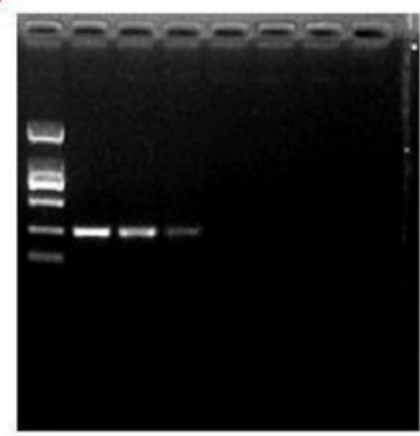

B
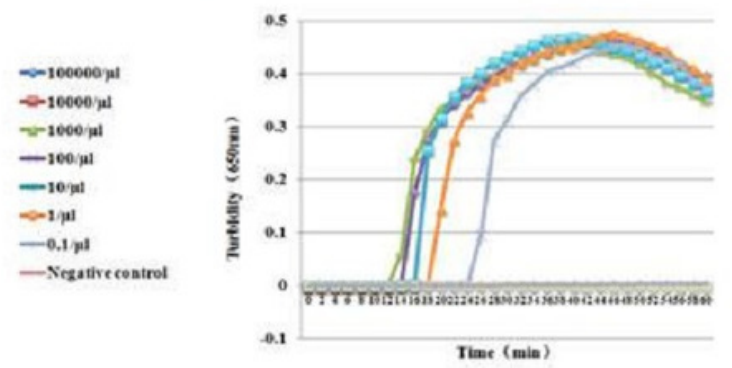

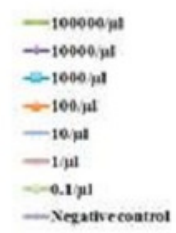

D

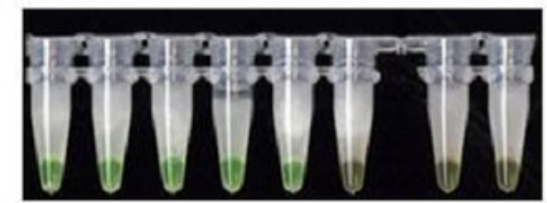

F

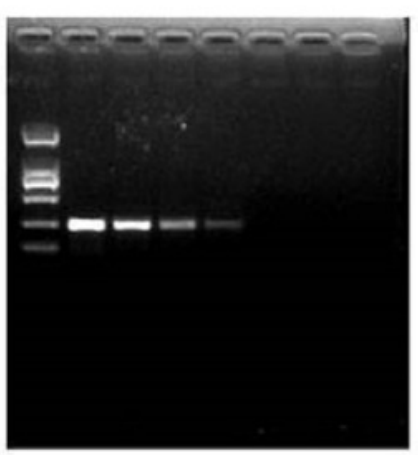


Figure 5

Fig.5 Detection of clinical specimens by RT-LAMP assay.

A: Results were analyzed using the LA-320C turbidimeter: JZGT-2, JZTH-5, JZGT-1, JZLH-4 and JZHS-5 weredeterminedtobepositive in the 23 clinical specimens. B: Results were analyzed through color changes: 1-8: JZHS-1, JZHS-2, JZGT-2, JZLH-1, JZLH-2, JZLH-3, JZTH-5, JZHS-3; 216: JZHS-4, JZGT-3, JZGT-4, JZGT-1, JZBZ-1, JZBZ-2, JZBZ-3, JZBZ-4; 17-23: JZLH-4, JZBZ-5, JZTH-1, JZHS-5, JZTH-2, JZTH-3, JZTH-4; 
$\mathbf{A}$

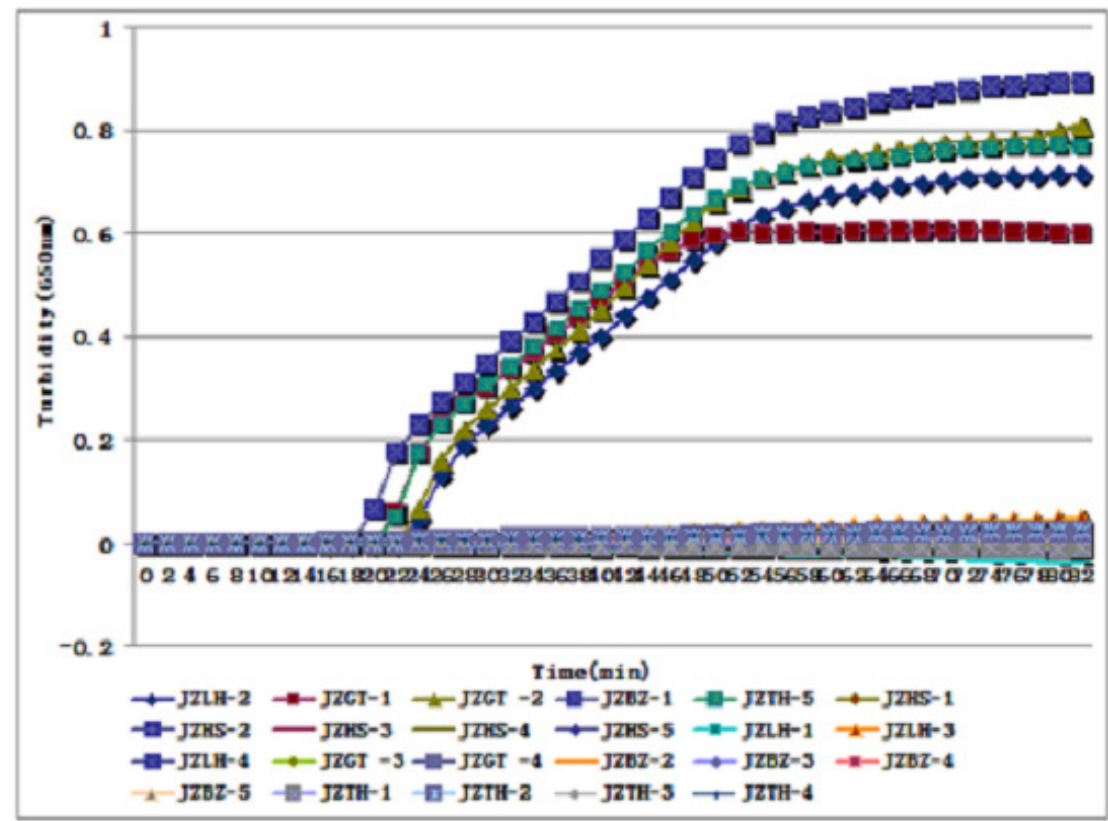

B

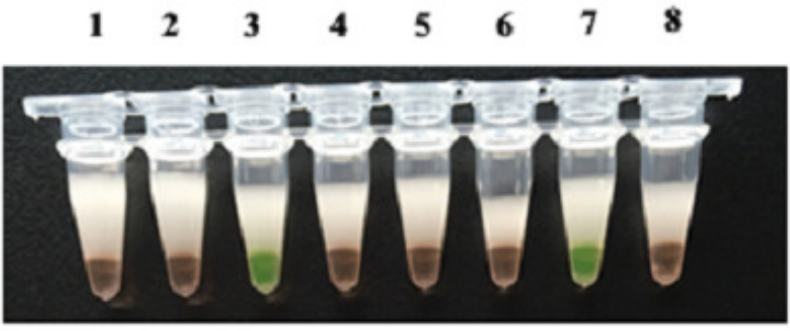

$\begin{array}{llllllll}9 & 10 & 11 & 12 & 13 & 14 & 15 & 16\end{array}$

$\begin{array}{lllllll}17 & 18 & 19 & 20 & 21 & 22 & 23\end{array}$

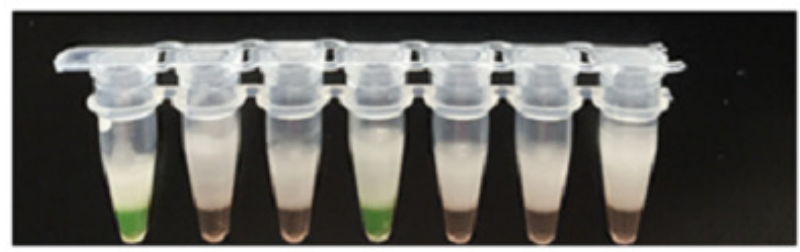

